\title{
Social dominance and familiarity modulate prosocial decision-making in rats
}

Michael Gachomba, Joan Esteve, Aroa Sanz Maroto, Cristina Márquez

Neural Circuits of Social Behavior Lab, Instituto de Neurociencias de Alicante (UMH-CSIC), Sant Joan d'Alacant, Spain

\section{INTRODUCTION}

- Prosocial behaviors - i.e., actions that benefit others - are pervasive in the animal kingdom, being essential for social bonding and cooperation. Several factors have been proposed to modulate prosocial behaviors, such as the familiarity of the partner and the social status of the interacting individuals. However, little is know about the behavioral and brain mechanisms that promote these interesting modulations. To target these questions, we investigate the effects of social context on prosocial behavior in rats, an animal model with rich social interactions and amenable of neural circuits manipulation.

- We previously showed that rats display prosocial choices in the absence of self-benefit (Márquez et al., 2015). Here we ask whether this kind decision-making is modulated by the familiarity and the social status of the interacting animals.

- In order to gain insights into the behavioral mechanisms underlying prosocial choices, we performed fine-grained quantification of social interactions, with subsecond resolution, using a custom-made automated tracking system for animal's body parts detection.

\section{METHODS}

\section{PROSOCIAL CHOICE TASK}

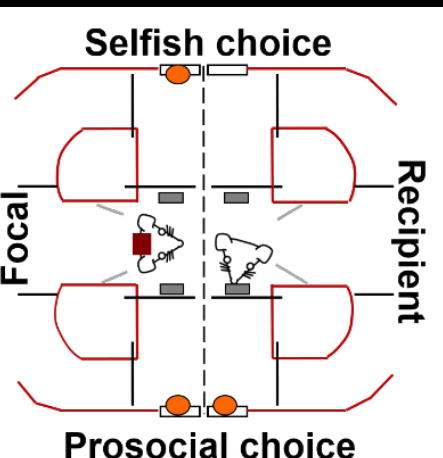

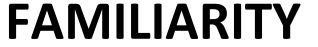

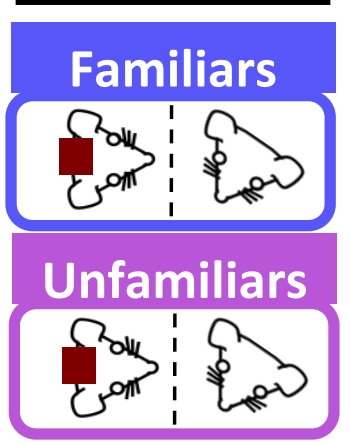

SOCIAL DOMINANCE

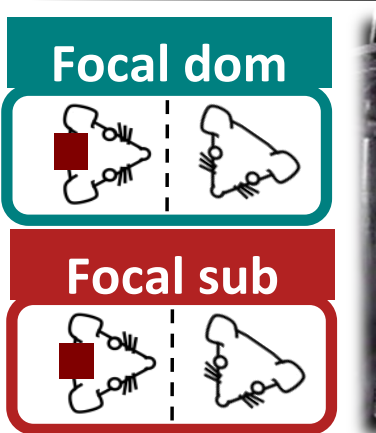

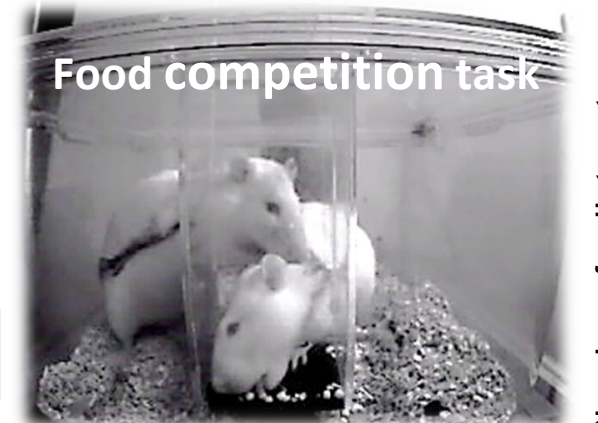

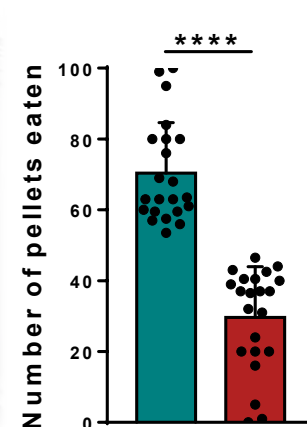

POSE ESTIMATION

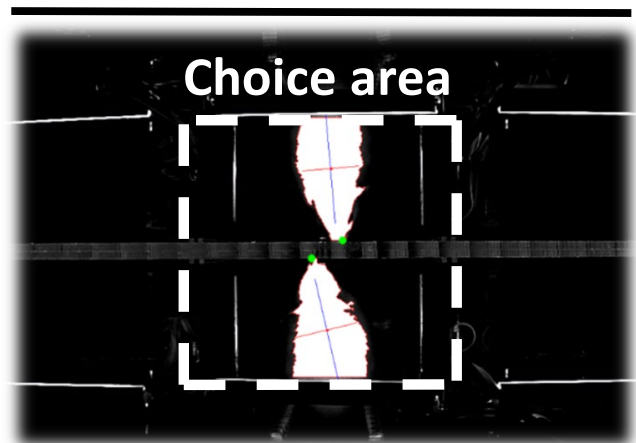

Prosocial choice task: in each trial, the decision-maker animal (focal) can decide to provide or not to provide food to a conspecific (recipient). Familiarity: pairs of cagemates and non-cagemates are tested in parallel for the choice task. Social dominance: social status is identified within each pair of cagemates through a food competition task (Costa et al., in preparation). Subsequently, animals perform the choice task with the focal being either the dominant or the submissive of the pair. Pose estimation: nose and centroid of the interacting animals are tracked in each trial prior to decision, over the first 2 days of the choice task.

\section{RESULTS}

Although FAMILIARITY has minor impact on choice, there are more selfish animals among unfamiliar pairs
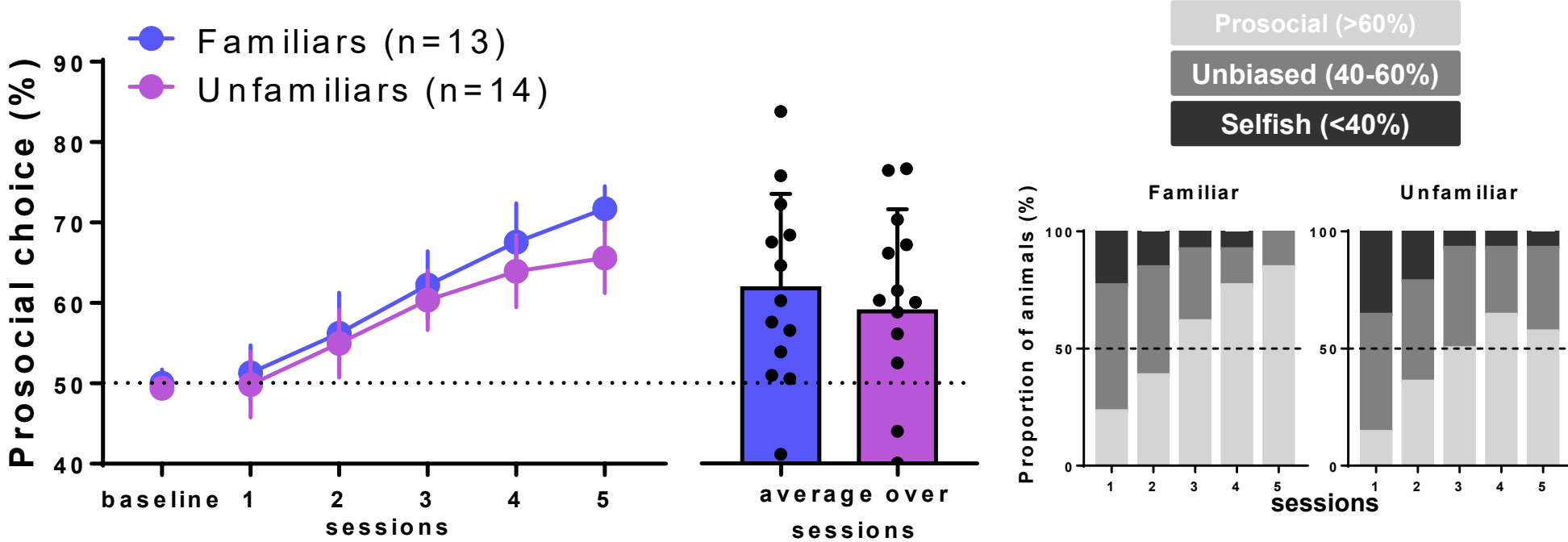

\section{SOCIAL DOMINANCE is a potent modulator of prosocial choice}

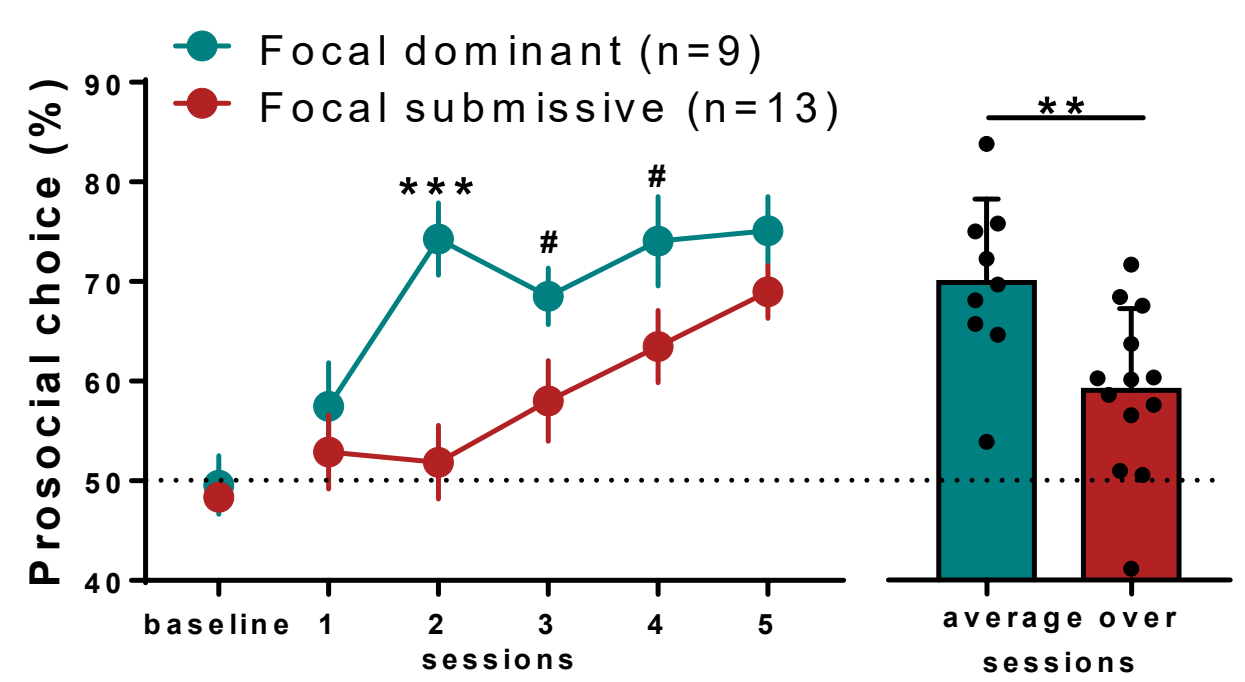

Evolution of prosocial choice preference over 5 daily consecutive sessions (line graphs). 'Baseline' indicates the preference of the focal animal averaged across the last 2 days of individual training. Bar graphs represent prosocial preference averaged across the 5 sessions

SOCIAL DOMINANCE affects the quality and not the quantity of social interactions
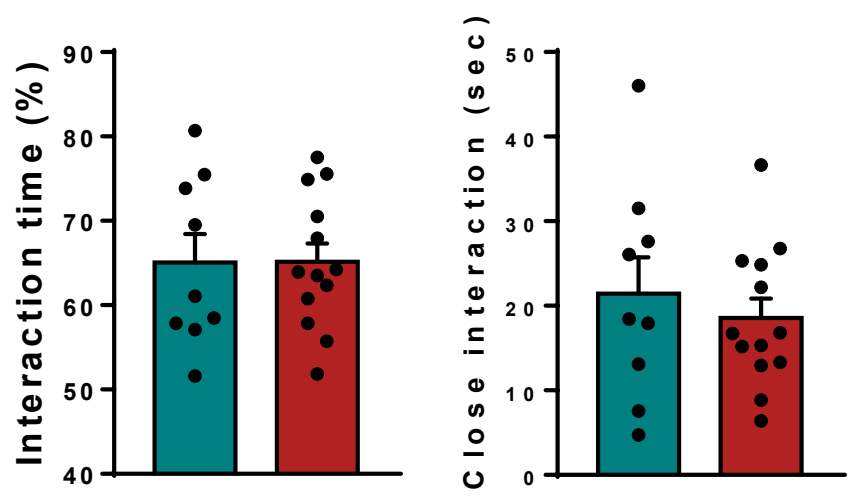

Social status doesn't affect the amount of time spent interacting and making direct contacts
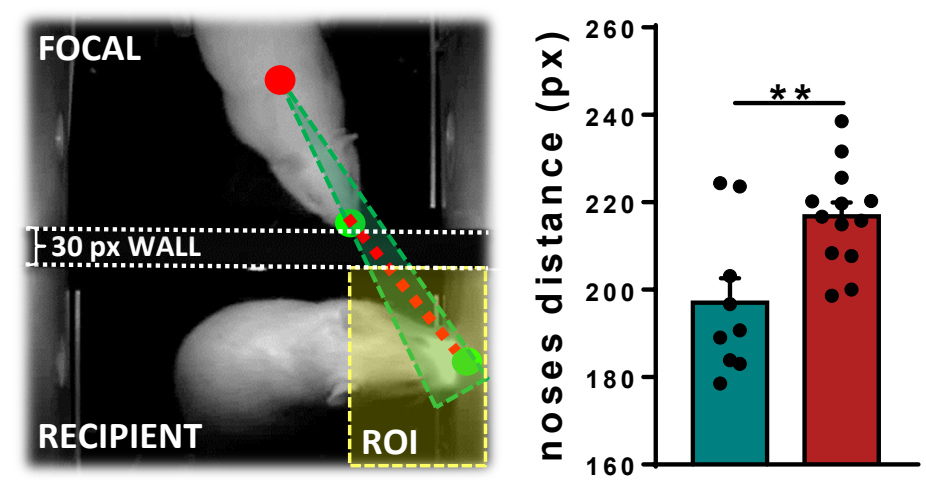
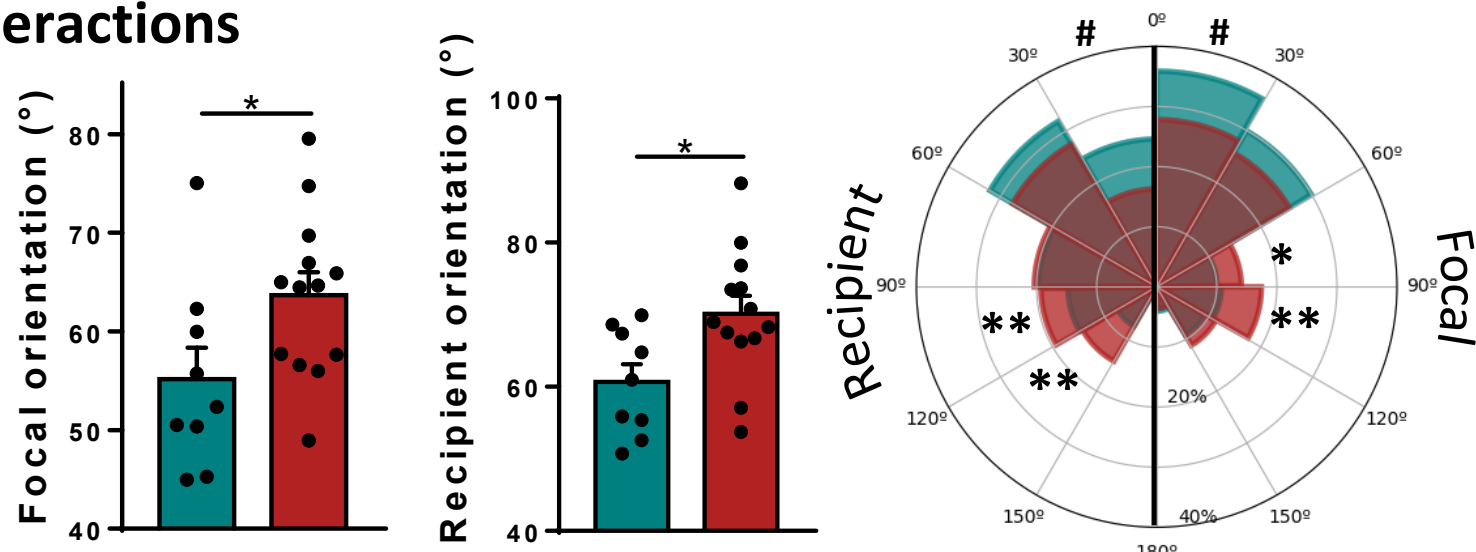

When the decision-maker is dominant, the two animals show more proximal social interactions and are more oriented to each others prior to decision. Radar plot shows the percentage of time spent by focal and recipient animal in each $30^{\circ}$-range orientation angle, when the recipient rat displays food-seeking behavior

Differences in the dynamics of social interactions emerge in the early moments of a trial, revealing patterns that may promote prosociality in rats

Focal dominant (prosocial trials) $\quad$ Focal submissive (prosocial trials)
Focal dominant (selfish trials)

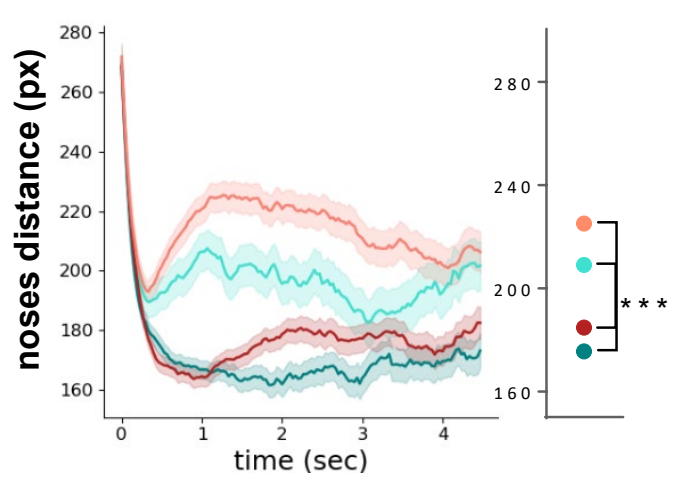

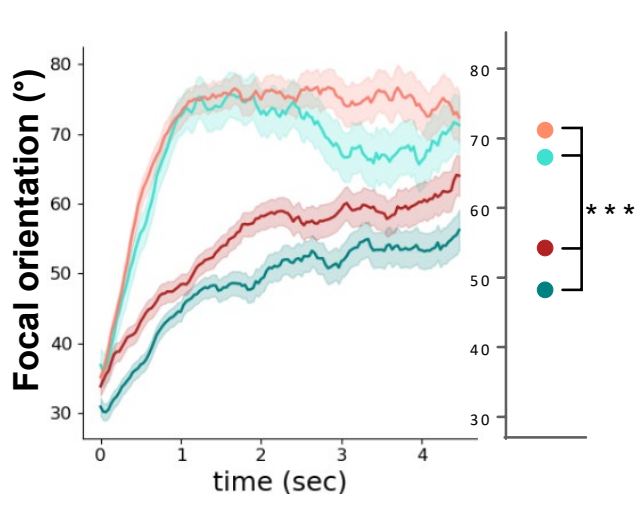

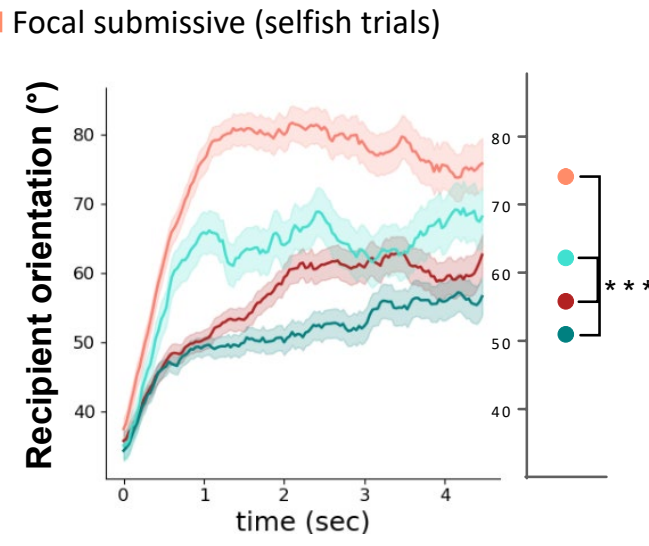

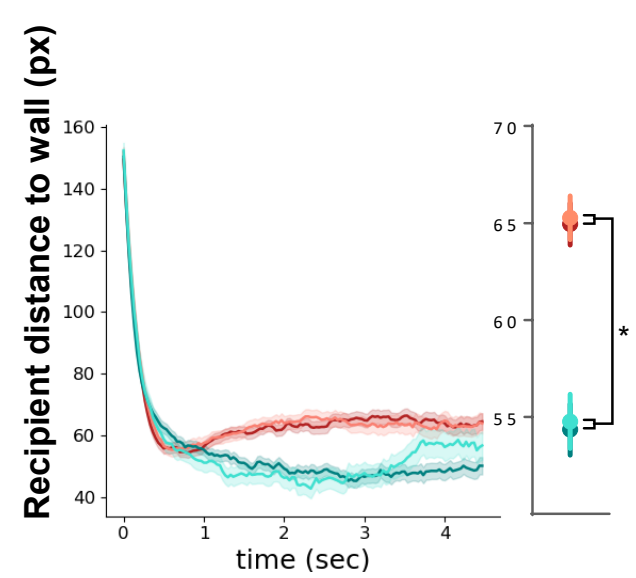

Submissive Recipients stay closer to the wall, both in prosocial and selfish trials

\section{CONCLUSIONS}

- Familiarity marginally modulates prosocial decision-making in rats

- Social dominance is a potent modulator of prosocial decision-making in rats, being dominants more prosocial

- Social dominance modulate the quality of social interactions, with increased prosociality accompanied by more proximal interactions and increased gazing in the moments before the decision 